\title{
Synthesis and Antioxidant Activity of Caffeic Acid Derivatives
}

\author{
Katarzyna Sidoryk ${ }^{1, *(1)}$, Anna Jaromin ${ }^{2}$, Nina Filipczak ${ }^{2}$, Piotr Cmoch $^{1}$ and Marcin Cybulski ${ }^{1}$ \\ 1 Pharmaceutical Research Institute, 8 Rydygiera Street, 01-793 Warsaw, Poland; p.cmoch@ifarm.eu (P.C.); \\ m.cybulski@ifarm.eu (M.C.) \\ 2 Department of Lipids and Liposomes, Faculty of Biotechnology, University of Wroclaw, \\ 14a Joliot-Curie Street, 50-383 Wroclaw, Poland; anna.jaromin@uwr.edu.pl (A.J.); \\ nina.filipczak@uwr.edu.pl (N.F.) \\ * Correspondence: k.sidoryk@ifarm.eu; Tel.: +48-22-456-39-28
}

Received: 1 August 2018; Accepted: 28 August 2018; Published: 30 August 2018

\begin{abstract}
A series of caffeic acid derivatives were synthesized via a modified Wittig reaction which is a very important tool in organic chemistry for the construction of unsaturated carbon-carbon bonds. All reactions were performed in water medium at $90{ }^{\circ} \mathrm{C}$. The aqueous Wittig reaction worked best when one unprotected hydroxyl group was present in the phenyl ring. The olefinations in the aqueous conditions were also conducted with good yields in the presence of two unprotected hydroxyl groups. When the number of the hydroxyl groups was increased to three, the reaction yields were worse, and the derivatives 12, 13, and 18 were obtained with $74 \%, 37 \%$, and 70\% yields, respectively. Nevertheless, the Wittig reaction using water as the essential medium is an elegant one-pot synthesis and a greener method, which can be a safe alternative for implementation in organic chemistry. The obtained compounds were tested for their antioxidant activity, and 12, 13, and 18 showed the highest activities. Moreover, all synthesized compounds displayed no cytotoxicity, and can therefore be used in the pharmaceutical or cosmetic industry.
\end{abstract}

Keywords: phenolic acids; derivatives of caffeic acid; Wittig reaction; water medium; antioxidant activity

\section{Introduction}

Caffeic acid (CA) is a natural phenolic acid which is synthesized by plants as a secondary metabolite. CA and its natural and synthetic derivatives show potent antioxidant activity, even in low concentrations. Moreover, it has been proved in many biological investigations that caffeic acid and its analogues also display anti-inflammatory, antibacterial, antiviral, and antitumor activities [1-5]. Recent investigations have demonstrated that caffeate esters, especially methyl caffeate, display sucrase and maltase inhibition [6-8].

Various biological studies on the phenolic acid molecules which have been carried out over the years have revealed that their varied biological activities stem from their antioxidant properties $[9,10]$. The antioxidant activity of phenolic acids follows from their acclaimed capability to scavenge reactive oxygen species (ROS) which include radical and nonradical oxygen species, such as $\mathrm{O}_{2}{ }^{-}, \mathrm{HO}, \mathrm{NO}$, and $\mathrm{H}_{2} \mathrm{O}_{2}$ [11-13]. The latest studies have disclosed that the presence of free phenolic hydroxyl groups and their number and position in the phenyl ring are essential for the strength of the antioxidant activity, while their protection renders them inactive [14]. Furthermore, the type of spacer between the carboxylic group and the aromatic ring of the phenolic acid also markedly influences their antioxidant profile-derivatives with methylenic, ethylenic, and unsaturated chains display the highest activity $[15,16]$. 
Due to advantageous biological effects, phenolic acids and their derivatives have become an essential instrument for the prevention or treatment of many diseases, and they have therefore found wide application in the cosmetic and pharmaceutical industries $[17,18]$. Phenolic acids, most notably caffeic acid, are also important scaffolds for the synthesis of a variety of biologically active compounds [19].

Simple commercially available phenolic acids are usually obtained by means of their extraction from natural plant sources, as well as by a biotechnological process [20]; however, chemical synthesis also plays an important role in accessing pure phenolic acids. In general, there are two most important routes to obtaining $\alpha, \beta$-unsaturated systems [21-24]. The first approach uses a modified Wittig reaction, namely the Horner-Wadsworth-Emmons (HWE) reaction. The second exploits Knoevenagel and aldol condensations. Unfortunately, the straightforward application of these known methods to obtain different analogues of caffeic acid has drawbacks, such as the multiplicity of procedures, side reactions, high temperature, long reaction time, low yield, and low stereoselectivity (see Scheme 1) [7,14,25]. For example, the reference method of 4 synthesis yields only $12 \%$, and the synthetic protocol contains the problematic deprotection of 3 by its demethylation at $-78^{\circ} \mathrm{C}$ in the presence of aggressive boron tribromide. Besides this, an alternative procedure to the modified Wittig olefination, based on the aldol condensation of aldehyde 5 with acetone, requires a prolonged reaction time, up to four days, to afford 6 with a moderate $58 \%$ yield.

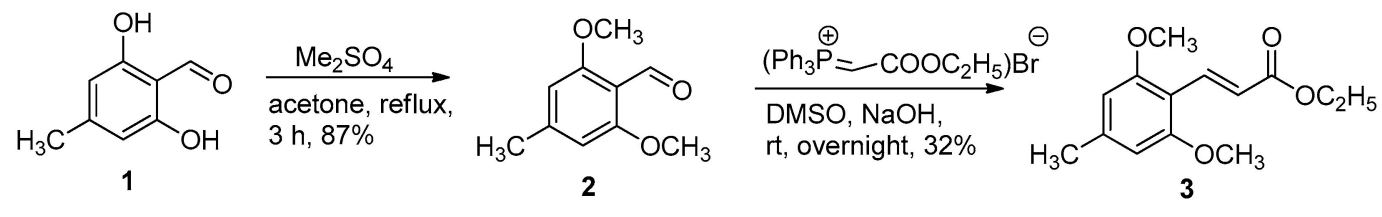<smiles>CCOC(=O)/C=C/c1c(OC)cc(C)cc1OC</smiles>

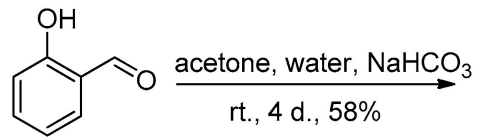

5<smiles>CC(=O)/C=C/c1ccccc1O</smiles>

6

Scheme 1. Examples of caffeic acid analogues' preparation.

To improve the HWE reaction, a number of variations on the reaction conditions have been reported. These optimizations include, e.g., increasing the temperature or pressure, the presence of additives, irradiation with microwaves or light, and use of silica gel or ionic solvents [26-30]. The latest investigation demonstrated that the HWE reaction could be conducted in water, which appeared to be an efficient medium, particularly for aromatic aldehydes [31]. Although the starting material and reaction products were very poorly soluble in water, the rate of reactions was fast. Moreover, the high yields of $80-98 \%$, short olefinations time, and very high $E / Z$-isomeric ratios in the olefination products prompted a detailed study of water as the essential medium in modified Wittig reactions [21,31,32]. While investigating the HWE reaction, the Bergdahl group demonstrated in one experiment that the water medium can be very effective for the $p$-hydroxybenzaldehyde substrate, having an unprotected phenolic group (Scheme 2) [31,32]. The appropriate cinnamate product 8 was obtained with a $92 \%$ yield, contrary to the same product in refluxing dichloromethane (DCM) which provided 8 with only an $8 \%$ yield [33]. 
<smiles>COC(=O)/C=C/c1ccc(O)cc1</smiles>

Scheme 2. A Wittig reaction of $p$-benzaldehyde with ylide in water.

This literature example encouraged us to study the HWE reaction in water using various aromatic aldehydes containing one or more unprotected hydroxyl groups in order to obtain a group of caffeic acid derivatives for biological evaluation. Moreover, the prospect of developing a synthetic method in water as the medium, to obtain the designed structures, seemed to be attractive because of mild reaction conditions and the ease of manipulation. The most important advantage of the investigated procedure, in comparison to the methods with a protected hydroxyl group, was the elimination of environmentally unsafe reagents (e.g., $\mathrm{BBr}_{3}$ ) and harsh reaction conditions.

Herein, we report a broad application of the HWE reaction in water to obtain different caffeate derivatives. Various aromatic aldehydes with the hydroxyl group in diverse positions of the aromatic ring ( $p$-, $m-, o$-benzaldehydes) or with additional hydroxyl groups (two or three hydroxyl groups in the aromatic ring), as well as different ylides, were verified as reaction substrates. Finally, the synthesized caffeic acid derivatives were tested for their antioxidant activity and cytotoxic effects on normal human dermal fibroblasts.

\section{Results and Discussion}

\subsection{Chemistry}

Inspired by the Bergdahl reports [31,32], we have investigated different aromatic aldehydes with unprotected hydroxyl groups (Table 1) in HWE reaction conditions. Although all the starting materials and reaction products were poorly soluble in water, the reaction yields were generally high. The presence of only one hydroxyl unprotected group in the substrate aromatic ring resulted in transformations ranging from 82 to $97 \%$ (Table 1, entries 1, 2, 3, 7 and 8, 9, 10, 14). However, for the $\mathrm{Ph}_{3} \mathrm{PCHCOCH}_{3}$ ylide substrate, a longer time was required to complete the reaction (Table 1, entries 8 , 9, and 10). The $E$ isomers were isolated as the major products.

The reaction of aromatic aldehydes with two unprotected hydroxyl groups in the aromatic ring resulted in favorable yields. Derivatives $\mathbf{1 1}$ and $\mathbf{1 7}$ were achieved with yields of $86 \%$ and $78 \%$, respectively. In contrast, the modified Wittig protocol worked very well for 4'-hydroxy-3-metoxybenzaldehyde (Table 1, entries 7 and 14). The reactions with both ylides I and II in water for $30 \mathrm{~min}$ at $90{ }^{\circ} \mathrm{C}$ led to $\mathbf{1 4}$ and $\mathbf{2 0}$ with $99 \%$ and $98 \%$ yields, respectively.

The introduction of a third hydroxyl group to the aromatic ring led to poorer results (Table 1 , entries 5, 6, 12, and 13). Compounds 12 and 18 were obtained with moderate $74 \%$ and $70 \%$ yields, respectively, in $1-0.5 \mathrm{~h}$ at $90^{\circ} \mathrm{C}$, while the prolonged reaction time caused the decomposition of the substrates. Another cinnamate product 13 was obtained with just a $37 \%$ yield. This resulted from the difficulties in isolating and purifying $\mathbf{1 3}$ from a much more impure crude material. The reaction of 2,4,5-trihydroxybenzaldehyde with the $\mathrm{Ph}_{3} \mathrm{PCHCOCH}_{3}$ ylide in water did not give product 19 , even though the reaction time was extended to $5 \mathrm{~h}$. Generally, a greater number of hydroxyl electron-donating groups in the benzaldehyde aromatic ring decreased the effectiveness of the conversion. This is consistent with the reported findings stating that electron-donating groups reduce the rate of the HWE reaction in methanol [31,32]. 
Table 1. The Horner-Wadsworth-Emmons (HWE) reaction of different aromatic aldehydes in water.

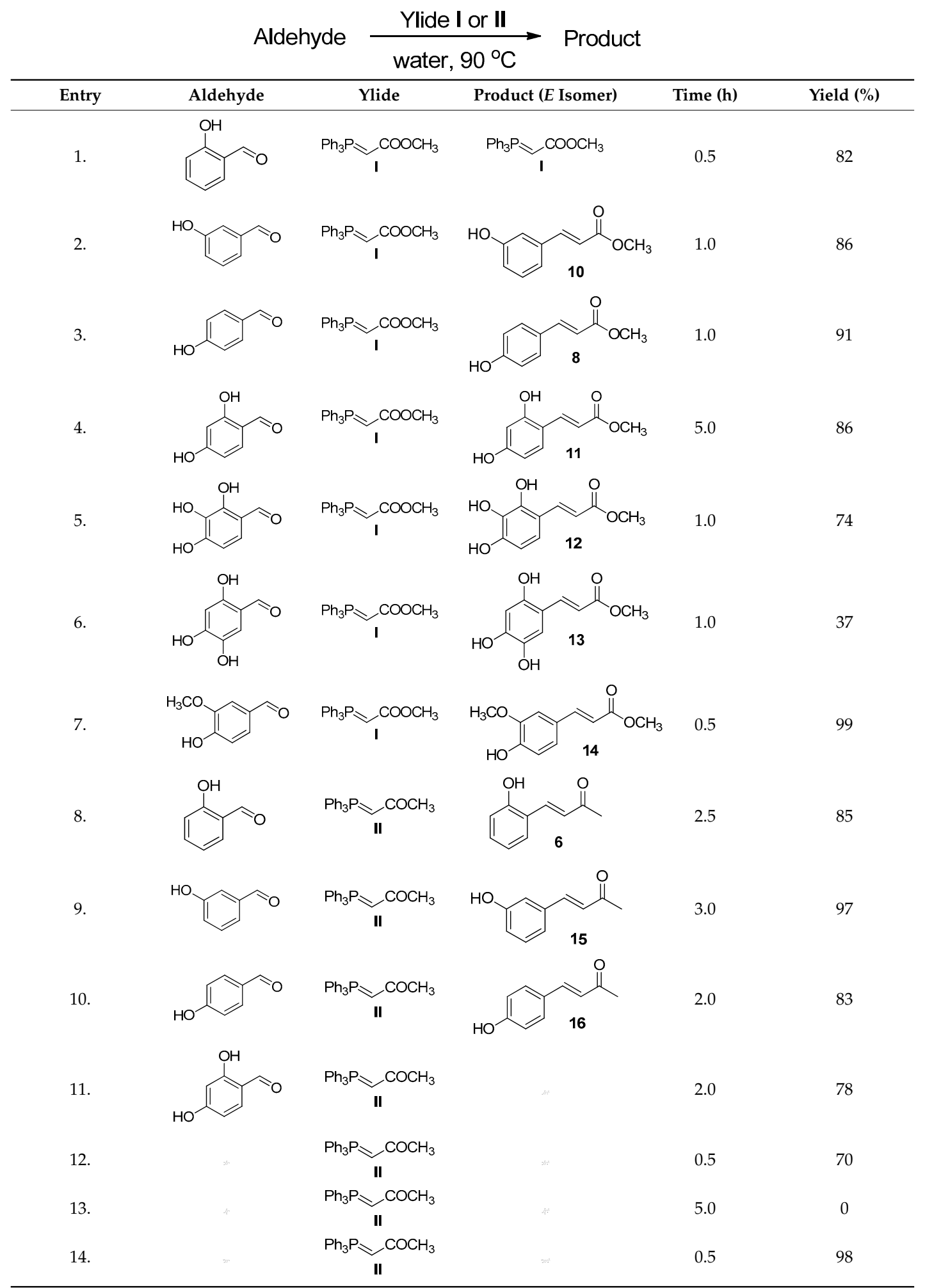

The structures of all compounds were confirmed by a detailed ${ }^{1} \mathrm{H}$ and ${ }^{13} \mathrm{C}-\mathrm{NMR}$ analysis (Section 3 and Supplementary Materials), as well as by HRMS experiments. In all examples the modified Wittig conditions generated the $E$-product that corresponded to the reference observation [31,32]. Our study proved that water was an effective medium for the HWE reaction. The one-step procedure provided 
products with high selectivity and good yields and its application for the synthesis of different phenolic compounds turned out to be easy, fast, and environmentally friendly.

\subsection{Biological Activity}

\subsubsection{Antioxidant Activity of CA Derivatives}

The antioxidant effect of CA and its derivatives was tested against lipid peroxidation in the $\mathrm{o} / \mathrm{w}$ emulsion model (Table 2). The high antioxidant activity of 12, 13, and 18 manifested in the highest protection against the induced oxidation results from the presence of three hydroxyl groups causing its low lipophilicity as suggested by calculated $\log P_{\mathrm{o} / \mathrm{w}}$ values. These results are generally in agreement with the radical scavenging activities determined by using a DPPH method. The DPPH assay relies on the measurement of discoloration, the effect of the reduction of a DPPH free radical caused by an antioxidant. Thus, lower $\mathrm{IC}_{50}$ values for $\mathbf{1 2}$ and $\mathbf{1 8}$ from the DPPH test express their high antioxidant activities. However, it seems that the presence of one $-\mathrm{OH}$ group is not sufficient to exert such an activity and 9, 10, 8, 6, 15, and 16 have no scavenging ability.

Table 2. $\log P_{\mathrm{o} / \mathrm{w}}$, and the antioxidant activity of the tested compounds.

\begin{tabular}{|c|c|c|c|}
\hline Compound & $\log P_{\mathrm{o} / \mathrm{w}}{ }^{\mathrm{a}}$ & $\begin{array}{l}\text { Inhibition of Oxidation } \\
\text { of o/w Emulsion }{ }^{b}\end{array}$ & DPPH IC ${ }_{50}(\mu \mathrm{M})$ \\
\hline CA & 0.93 & $26.1 \pm 2.9$ & 32.2 \\
\hline 9 & 1.82 & $2.6 \pm 1.5$ & n.a. ${ }^{\mathrm{c}}$ \\
\hline 10 & 1.78 & $18.4 \pm 2.2$ & n.a. ${ }^{\mathrm{c}}$ \\
\hline 8 & 1.81 & $22.7 \pm 6.4$ & n.a. ${ }^{\mathrm{c}}$ \\
\hline 11 & 1.31 & $33.7 \pm 6.5$ & 1015.9 \\
\hline 12 & 0.99 & $55.5 \pm 2$ & 18.6 \\
\hline 13 & 0.95 & $38.6 \pm 2.3$ & 53.3 \\
\hline 14 & 1.76 & $14.8 \pm 2$ & 58.9 \\
\hline 6 & 1.94 & n.a. ${ }^{\mathrm{c}}$ & n.a. ${ }^{\mathrm{d}}$ \\
\hline 15 & 1.82 & n.a. ${ }^{\mathrm{c}}$ & n.a. ${ }^{\mathrm{d}}$ \\
\hline 16 & 1.85 & $6.8 \pm 4.4$ & n.a. ${ }^{\mathrm{d}}$ \\
\hline 17 & 1.49 & $4.6 \pm 1.3$ & 688.2 \\
\hline 18 & 1.05 & $28.6 \pm 4$ & 16.8 \\
\hline 20 & 1.86 & $7.9 \pm 2.1$ & 61.8 \\
\hline
\end{tabular}

a The prediction of $\log P_{\mathrm{o} / \mathrm{w}}$ was performed using the SwissADME program; ${ }^{\mathrm{b}}$ determined at $100 \mu \mathrm{M} ;{ }^{\mathrm{c}}$ no protection detected; ${ }^{\mathrm{d}} \mathrm{IC}_{50}>5 \mathrm{mM}$. CA, caffeic acid.

\subsubsection{Effects of CA Derivatives on NHDF Cell Viability}

Having proven the potent antioxidant activity of the synthesized compounds and in the light of encouraging reports concerning significant protection against UV-radiation-induced skin damage [34], reduction of UV-induced skin aging [35], and anti-wrinkling activity [36], we went on to determine their preliminary cytotoxicity against skin cells.

In this study we examined the biocompatibility of the synthesized compounds on the normal human dermal fibroblast (NHDF) cell line by MTT assay. Figure 1A,B show the viability of NHDF incubated with compounds at concentrations of $12.5-250 \mu \mathrm{M}$. All compounds did not affect the viability of human cells in vitro for 24 and $48 \mathrm{~h}$, except for $\mathbf{1 3}$ and $\mathbf{2 0}$ at the highest concentration ( $24 \mathrm{~h}$ ). However, in the case of these two compounds, this effect after $48 \mathrm{~h}$ was not clearly noticeable, probably due to their selective pumping out. This observation is very interesting taking into account the potential use of these active agents as components of skin or hair care products. However, in order to confirm their cosmeceutical potential and establish their bioavailability after topical application, several additional evaluations are needed, such as skin irritation and sensitization tests, development of optimal formulations, and evaluation of their effectiveness, as well as skin permeation studies. 


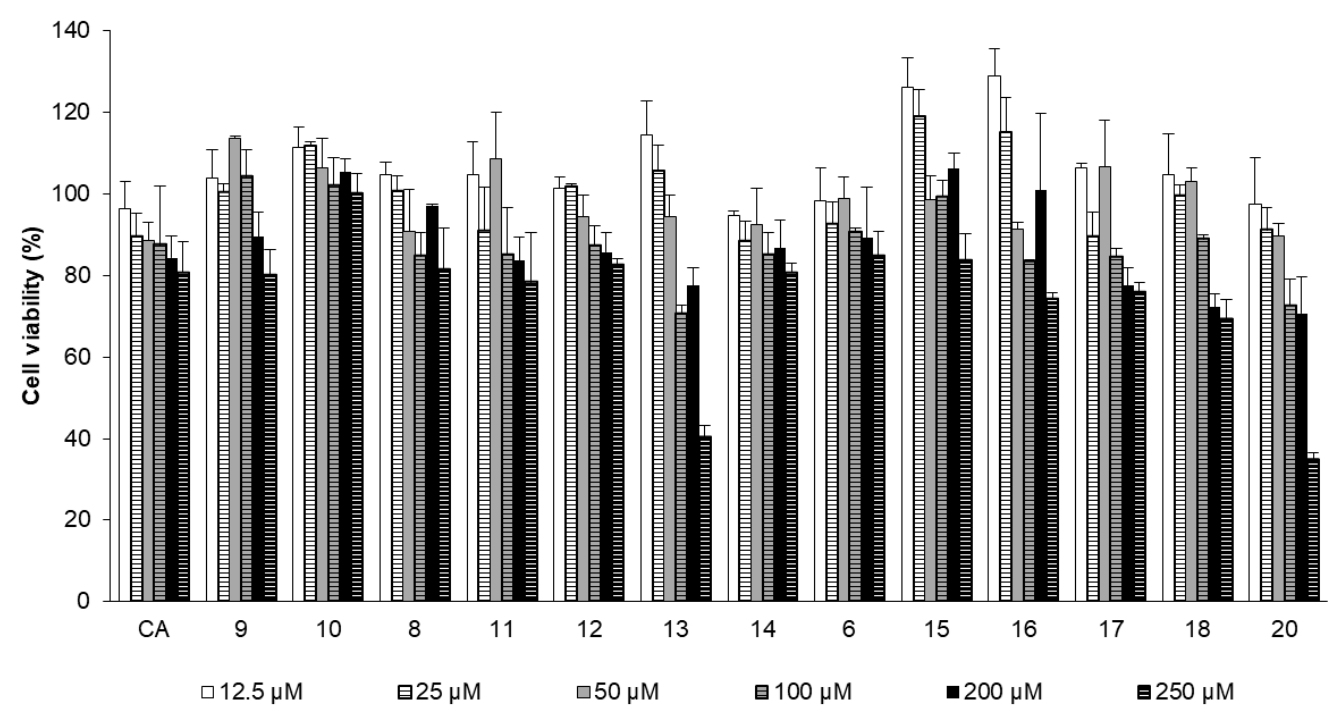

(A)

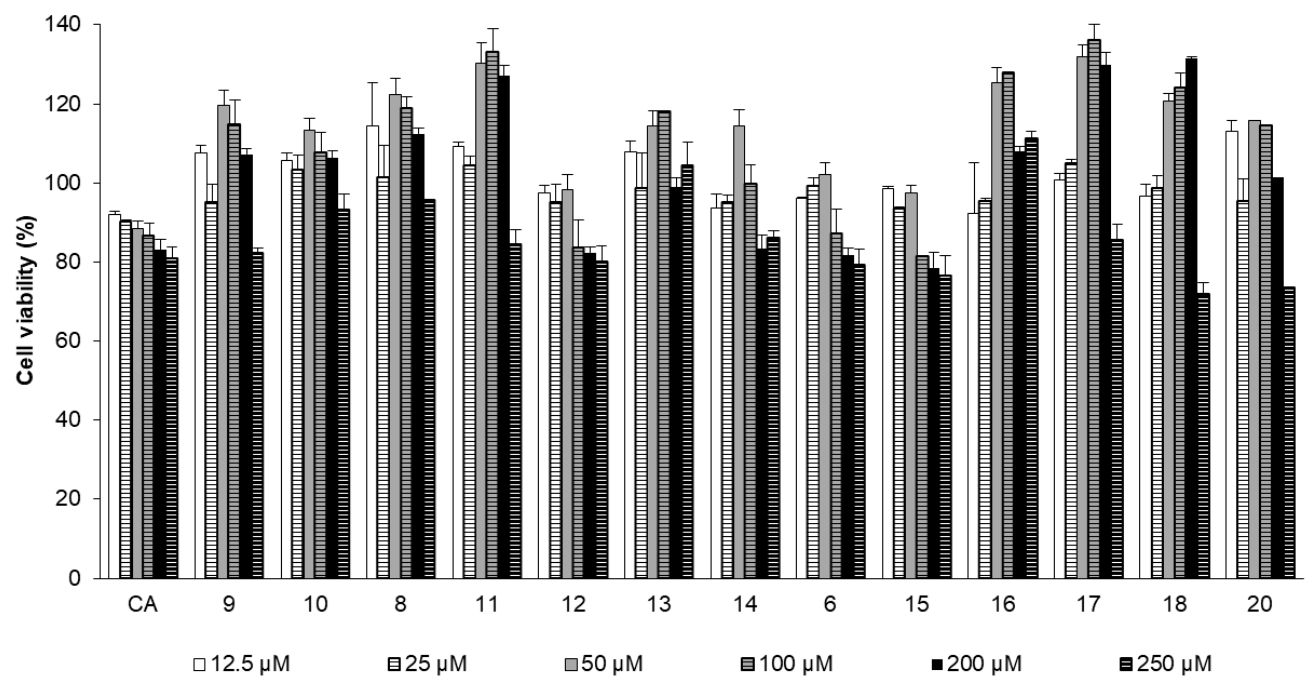

(B)

Figure 1. Cell viability in normal human dermal fibroblast (NHDF) cells after $24 \mathrm{~h} \mathrm{(A)}$ and $48 \mathrm{~h}$ (B) exposure to different concentrations of $\mathrm{CA}$ and its derivatives. The cell viability was evaluated with MTT assay and the results were presented as the percentage of the control groups. The data are presented as the mean standard deviation (SD) of three independent experiments.

\section{Experimental}

\subsection{Chemistry}

\subsubsection{General Materials and Methods}

All reagent and solvents were purchased from common commercial suppliers without further purification. For monitoring the reaction progress, Merck DC-Alufolien Kieselgel $60 \mathrm{~F}_{25}$ TLC plates were used. The column chromatography was performed on Merck silica gel 60 230-400 mesh (Merck Group, Darmstadt, Germany). Melting points were measured on a Mettler Toledo MP90 apparatus (Mettler-Toledo GmbH, Greifensee, Switzerland) and were uncorrected. NMR spectra were recorded on Varian VNMRS 500 and Varian VNMRS 600 (Varian Medical Systems Inc., Palo Alto, CA, USA) spectrometers (at $298 \mathrm{~K}$ ) in $\mathrm{CDCl}_{3}$ or $\mathrm{CD}_{3} \mathrm{OD}$ using TMS as an internal standard. 
The ESI-MS spectra were recorded on a PE Biosystems Mariner mass spectrometer (Thermo Fisher Scientific, Waltham, MA, USA).

\subsubsection{General Method for the HWE Reaction in Water}

A suspension of an appropriate aromatic aldehyde (1 eq.), and ylide I or II (1.3-1.5 eq.) in water $(4-10 \mathrm{~mL})$ was stirred at $90{ }^{\circ} \mathrm{C}$ for $0.5-24 \mathrm{~h}$. Next, the heterogeneous reaction mixture was cooled to room temperature, and the aqueous phase was extracted with DCM $(3 \times 10 \mathrm{~mL})$. The solvent was evaporated under diminished pressure. Column chromatography (hexane-ethyl acetate, or chloroform-methanol) of the residue gave the E-alkene as the major product.

3-(2.'-Hydroxyphenyl)-(E)-propenoic acid methyl ester (9). 9 was obtained from 2-hydroxybenzaldehyde $(0.82 \mathrm{mmol}, 85 \mu \mathrm{L})$ and (methoxycarbonylmethyl)triphenylphosphine $(1.23 \mathrm{mmol}, 410 \mathrm{mg})$. Reaction was in water $(4.0 \mathrm{~mL})$ at $90{ }^{\circ} \mathrm{C}$ for $0.5 \mathrm{~h}$. The crude product 9 was purified by column chromatography (EtOAc/hexane, $10: 1 \rightarrow 3: 1$ ) to give $82 \%$ (120 mg) of 9 as a white solid. mp. $118-119^{\circ} \mathrm{C}$ (lit. mp.: $119-120^{\circ} \mathrm{C}$ [37]); NMR consistent with literature data [37]; HR-MS (ES) calcd. for $\mathrm{C}_{10} \mathrm{H}_{10} \mathrm{O}_{3}(\mathrm{M})^{-}$: 177.0552. Found: 177.0551.

3-(3'-Hydroxyphenyl)-(E)-propenoic acid methyl ester (10). $\mathbf{1 0}$ was obtained from 3-hydroxybenzaldehyde $(0.82 \mathrm{mmol}, 100 \mathrm{mg}$ ) and (methoxycarbonylmethyl)triphenylphosphine $(1.23 \mathrm{mmol}, 410 \mathrm{mg})$. Reaction was in water $(4.0 \mathrm{~mL})$ at $90{ }^{\circ} \mathrm{C}$ for $1 \mathrm{~h}$. The crude product 10 was purified by column chromatography (EtOAc/hexane, 10:1 $\rightarrow 3: 1)$ to give $86 \%$ (126 mg) of $\mathbf{1 0}$ as a white solid. mp. $78-81{ }^{\circ} \mathrm{C}$ (lit. mp.: 79-81 ${ }^{\circ} \mathrm{C}$ [37]); NMR consistent with literature data [38]; HR-MS (ES) calcd. for $\mathrm{C}_{10} \mathrm{H}_{10} \mathrm{O}_{3}(\mathrm{M})^{-}$: 177.0552. Found: 177.0555.

3-(4'-Hydroxyphenyl)-(E)-propenoic acid methyl ester (8). $\mathbf{8}$ was obtained from 4-hydroxybenzaldehyde $(0.65 \mathrm{mmol}, 80 \mathrm{mg})$ and (methoxycarbonylmethyl)triphenylphosphine $(0.98 \mathrm{mmol}, 328 \mathrm{mg})$. Reaction was in water $(4.0 \mathrm{~mL})$ at $90{ }^{\circ} \mathrm{C}$ for $1 \mathrm{~h}$. The crude product 8 was purified by column chromatography (EtOAc/hexane, 10:1 $\rightarrow 3: 1$ ) to give $91 \%$ (106 $\mathrm{mg}$ ) of 8 as a white solid. mp. $132-134{ }^{\circ} \mathrm{C}$ (Lit. mp. $128-133{ }^{\circ} \mathrm{C}$ [32]); NMR consistent with literature data [33]; HR-MS (ES) calcd. for $\mathrm{C}_{10} \mathrm{H}_{10} \mathrm{O}_{3}(\mathrm{M})^{-}$: 177.0552. Found: 177.0552.

3-(2', $4^{\prime}$-dihydroxyphenyl)-(E)-propenoic acid methyl ester (11). $\mathbf{1 1}$ was obtained from 2,4-dihydroxybenzaldehyde ( $0.72 \mathrm{mmol}, 100 \mathrm{mg}$ ) and (methoxycarbonylmethyl)triphenylphosphine $(1.08 \mathrm{mmol}, 363 \mathrm{mg})$. Reaction was in water $(5.0 \mathrm{~mL})$ at $90{ }^{\circ} \mathrm{C}$ for $5 \mathrm{~h}$. The crude product 11 was purified by column chromatography (EtOAc/hexane, 1:1) to give $86 \%(120 \mathrm{mg})$ of $\mathbf{1 1}$ as a white solid. mp. $158-160{ }^{\circ} \mathrm{C} .{ }^{1} \mathrm{H}-\mathrm{NMR}\left(500 \mathrm{MHz}, \mathrm{CDCl}_{3}+\mathrm{CD}_{3} \mathrm{OD}\right) \delta 7.93\left(\mathrm{~d}, 1 \mathrm{H}, \mathrm{CH}^{\beta}=\mathrm{CH}^{\alpha}, J=16 \mathrm{~Hz}\right)$, $7.31(\mathrm{~d}$, aromatic, $1 \mathrm{H}, J=8.5 \mathrm{~Hz}), 6.44\left(\mathrm{~d}, 1 \mathrm{H}, \mathrm{CH}^{\beta}=\mathrm{CH}^{\alpha}, J=16 \mathrm{~Hz}\right), 6.35(\mathrm{dd}$, aromatic, $1 \mathrm{H}, J=2 \mathrm{~Hz}$, $J=8.5 \mathrm{~Hz}), 6.31(\mathrm{~d}$, aromatic, $1 \mathrm{H}, J=2 \mathrm{~Hz}), 3.77\left(\mathrm{~s}, \mathrm{CH}_{3}, 3 \mathrm{H}\right) ;{ }^{13} \mathrm{C}-\mathrm{NMR}\left(125 \mathrm{MHz}, \mathrm{CDCl}_{3}+\mathrm{CD}_{3} \mathrm{OD}\right) \delta$ 169.2, 160.1, 158.1, 141.2, 130.4, 113.8, 113.5, 107.7, 102.4, 51.2; HR-MS (ES) calcd. for $\mathrm{C}_{10} \mathrm{H}_{10} \mathrm{O}_{4}(\mathrm{M})^{-}$: 193.0501. Found: 193.0503.

3-(2', 3', $4^{\prime}$-trihydroxyphenyl)-(E)-propenoic acid methyl ester (12). 12 was obtained from 2,3,4-trihydroxybenzaldehyde (12.1 mmol, $1.86 \mathrm{~g}$ ) and (methoxycarbonylmethyl)triphenylphosphine $(15.73 \mathrm{mmol}, 5.26 \mathrm{~g})$. Reaction was in water $(65 \mathrm{~mL})$ at $90{ }^{\circ} \mathrm{C}$ for $1 \mathrm{~h}$. The crude product 12 was purified by column chromatography (chloroform/methanol, 10:1 $\rightarrow 11: 1)$ to give $74 \%$ (1.85 g) of $\mathbf{1 2}$

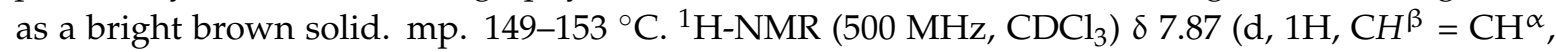
$J=16.1 \mathrm{~Hz}), 6.88(\mathrm{~d}$, aromatic, $1 \mathrm{H}, J=8.5 \mathrm{~Hz}), 6.43\left(\mathrm{~d}, 1 \mathrm{H}, \mathrm{CH}^{\beta}=\mathrm{CH}^{\alpha}, J=16.1 \mathrm{~Hz}\right), 6.35(\mathrm{~d}$, aromatic, $1 \mathrm{H}, J=8.5 \mathrm{~Hz}), 3.74\left(\mathrm{~s}, \mathrm{CH}_{3}, 3 \mathrm{H}\right) ;{ }^{13} \mathrm{C}-\mathrm{NMR}\left(125 \mathrm{MHz}, \mathrm{CDCl}_{3}\right) \delta 170.6,149.6,148.2,143.1,134.0,121.2$, 115.4, 114.5, 108.5, 51.8; HR-MS (ES) calcd. for $\mathrm{C}_{10} \mathrm{H}_{10} \mathrm{O}_{5}(\mathrm{M})^{-}$: 209.0450. Found: 209.0446.

3-(2', 4',5'-trihydroxyphenyl)-(E)-propenoic acid methyl ester (13). 13 was obtained from 2,4,5-trihydroxybenzaldehyde $(1.29 \mathrm{mmol}, 200 \mathrm{mg}$ ) and (methoxycarbonylmethyl)triphenylphosphine $(1.68 \mathrm{mmol}, 560 \mathrm{mg})$. Reaction was in water $(7 \mathrm{~mL})$ at $90{ }^{\circ} \mathrm{C}$ for $60 \mathrm{~min}$. The crude product 13 was purified by column chromatography (chloroform/methanol, 10:1 $\rightarrow 1: 1$ ) to give $37 \%$ (100 $\mathrm{mg}$ ) of $\mathbf{1 3}$ as a bright brown solid. mp. $150-152{ }^{\circ} \mathrm{C} .{ }^{1} \mathrm{H}-\mathrm{NMR}\left(600 \mathrm{MHz}, \mathrm{CD}_{3} \mathrm{OD}\right) \delta 7.91\left(\mathrm{~d}, 1 \mathrm{H}, \mathrm{CH}^{\beta}=\mathrm{CH}^{\alpha}\right.$, $J=16.2 \mathrm{~Hz}), 6.89(\mathrm{~s}$, aromatic, $1 \mathrm{H}), 6.34(\mathrm{~s}$, aromatic, $1 \mathrm{H}), 6.28\left(\mathrm{~d}, 1 \mathrm{H}, \mathrm{CH}^{\beta}=\mathrm{CH}^{\alpha}, J=16.2 \mathrm{~Hz}\right)$, 
$3.74\left(\mathrm{~s}, \mathrm{CH}_{3}, 3 \mathrm{H}\right) ;{ }^{13} \mathrm{C}-\mathrm{NMR}\left(150 \mathrm{MHz}, \mathrm{CD}_{3} \mathrm{OD}\right) \delta 170.6,152.9,150.8,142.4,139.8,114.6,113.7,113.4$, 104.2, 51.8; HR-MS (ES) calcd. for $\mathrm{C}_{10} \mathrm{H}_{10} \mathrm{O}_{5}(\mathrm{M})^{-}:$: 209.0450. Found: 209.0447.

3-(4'-hydroxy-3-metoxyphenyl)-(E)-propenoic acid methyl ester (14). 14 was obtained from 4-hydroxy-3-methoxybenzaldehyde $(0.65 \quad \mathrm{mmol}, 100 \mathrm{mg})$ and (methoxycarbonylmethyl)triphenylphosphine $(0.98 \mathrm{mmol}, 329 \mathrm{mg})$. Reaction was in water $(4 \mathrm{~mL})$ at $90{ }^{\circ} \mathrm{C}$ for $0.5 \mathrm{~h}$. The crude product 14 was purified by column chromatography (EtOAc/hexane, $5: 1 \rightarrow 1: 1)$ to give $99 \%\left(134 \mathrm{mg}\right.$ ) of $\mathbf{1 4}$ as a white solid. mp. $67-69{ }^{\circ} \mathrm{C}$ (lit. mp. 62-63 $\left.{ }^{\circ} \mathrm{C}[16]\right) .{ }^{1} \mathrm{H}-\mathrm{NMR}\left(500 \mathrm{MHz}, \mathrm{CDCl}_{3}\right) \delta 7.63\left(\mathrm{~d}, 1 \mathrm{H}, \mathrm{CH}^{\beta}=\mathrm{CH}^{\alpha}, J=16 \mathrm{~Hz}\right), 7.08(\mathrm{~d}$, aromatic, $1 \mathrm{H}$, $J=1.5 \mathrm{~Hz}), 7.07(\mathrm{~m}$, aromatic, $1 \mathrm{H}, J=1.5 \mathrm{~Hz}, J=2 \mathrm{~Hz}), 7.02(\mathrm{~m}$, aromatic, $1 \mathrm{H}, J=1.5 \mathrm{~Hz}), 6.27(\mathrm{~d}, 1 \mathrm{H}$, $\left.\mathrm{CH}^{\beta}=\mathrm{CH}^{\alpha}, J=16 \mathrm{~Hz}\right), 3.92\left(\mathrm{~s}, \mathrm{CH}_{3}, 3 \mathrm{H}\right), 3.79\left(\mathrm{~s}, \mathrm{CH}_{3}, 3 \mathrm{H}\right) ;{ }^{13} \mathrm{C}-\mathrm{NMR}\left(125 \mathrm{MHz}, \mathrm{CDCl}_{3}\right) \delta 167.7$, $147.9,146.7,144.9,126.9,123.0,115.1,114.7,109.3,55.9,51.5$; HR-MS (ES) calcd. for $\mathrm{C}_{11} \mathrm{H}_{12} \mathrm{O}_{4}(\mathrm{M})^{-}$: 207.0657. Found: 207.0654.

4-(2'-hydroxyphenyl)-3(E)-buten-2-one (6). 6 was obtained from 2-hydroxybenzaldehyde (0.82 mmol, $85 \mu \mathrm{L}$ ) and 1-(triphenylphosphoranylidene)-2-propanone (1.23 mmol, $391 \mathrm{mg})$. Reaction was in water $(4.0 \mathrm{~mL})$ at $90{ }^{\circ} \mathrm{C}$ for $2.5 \mathrm{~h}$. The crude product 6 was purified by column chromatography (EtOAc/hexane, $5: 1 \rightarrow 1: 1)$ to give $85 \%$ (112 mg) of 6 as a yellow solid. mp. $137-139{ }^{\circ} \mathrm{C}$ (lit. mp. 139-140 ${ }^{\circ} \mathrm{C}$ [25]); NMR consistent with literature data [25]; HR-MS (ES) calcd. for $\mathrm{C}_{10} \mathrm{H}_{10} \mathrm{O}_{2}(\mathrm{M})^{-}$: 161.0603. Found: 161.0604.

4-(3'-hydroxyphenyl)-3(E)-buten-2-one (15). 15 was obtained from 3-hydroxybenzaldehyde (0.82 mmol, $100 \mathrm{mg})$ and 1-(triphenylphosphoranylidene)-2-propanone (1.23 mmol, $391 \mathrm{mg})$. Reaction was in water $(4.0 \mathrm{~mL})$ at $90{ }^{\circ} \mathrm{C}$ for $3 \mathrm{~h}$. The crude product was purified by column chromatography (EtOAc/hexane, $5: 1 \rightarrow 1: 1)$ to give $97 \%\left(128 \mathrm{mg}\right.$ ) of $\mathbf{1 5}$ as a white solid. mp. $94-96{ }^{\circ} \mathrm{C}$ (lit. mp. $\left.96-97^{\circ} \mathrm{C}[21]\right) .{ }^{1} \mathrm{H}-\mathrm{NMR}\left(500 \mathrm{MHz}, \mathrm{CDCl}_{3}\right) \delta 7.50\left(\mathrm{~d}, 1 \mathrm{H}, \mathrm{CH}^{\beta}=\mathrm{CH}^{\alpha}, J=16.0 \mathrm{~Hz}\right)$, 7.28-7.24 (m, aromatic, $1 \mathrm{H}), 7.10-7.08(\mathrm{~m}$, aromatic, $2 \mathrm{H}), 6.94-6.92(\mathrm{~m}$, aromatic, $1 \mathrm{H}), 6.71\left(\mathrm{~d}, 1 \mathrm{H}, \mathrm{CH}^{\beta}\right.$ $\left.=\mathrm{CH}^{\alpha}, J=16.0 \mathrm{~Hz}\right), 2.40\left(\mathrm{~s}, \mathrm{CH}_{3}, 3 \mathrm{H}\right) ;{ }^{13} \mathrm{C}-\mathrm{NMR}\left(125 \mathrm{MHz}, \mathrm{CDCl}_{3}\right) \delta 199.7,156.5,144.2,135.7,130.2$, 127.1, 121.0, 118.1, 114.6, 27.4; HR-MS (ES) calcd. for $\mathrm{C}_{10} \mathrm{H}_{10} \mathrm{O}_{2}(\mathrm{M})^{-}$: 161.0603. Found: 161.0602.

4-(4'-hydroxyphenyl)-3(E)-buten-2-one (16). 16 was obtained from 4-hydroxybenzaldehyde (0.82 mmol, $100 \mathrm{mg}$ ) and 1-(triphenylphosphoranylidene)-2-propanone (1.23 mmol, $391 \mathrm{mg})$. Reaction was in water $(4.0 \mathrm{~mL})$ at $90{ }^{\circ} \mathrm{C}$ for $2 \mathrm{~h}$. The crude product was purified by column chromatography (EtOAc/hexane, $5: 1 \rightarrow 1: 1)$ to give $83 \%(110 \mathrm{mg})$ of $\mathbf{1 6}$ as a white solid. mp. 110-111 ${ }^{\circ} \mathrm{C}$ (lit. mp. $\left.111-113{ }^{\circ} \mathrm{C}[38]\right) .{ }^{1} \mathrm{H}-\mathrm{NMR}\left(500 \mathrm{MHz}, \mathrm{CDCl}_{3}\right) \delta 7.53\left(\mathrm{~d}, 1 \mathrm{H}, \mathrm{CH}^{\beta}=\mathrm{CH}^{\alpha}\right.$, $J=16.0 \mathrm{~Hz}), 7.45-7.43(\mathrm{~m}$, aromatic, $2 \mathrm{H}), 6.93-6.90(\mathrm{~m}$, aromatic, $2 \mathrm{H}), 6.62\left(\mathrm{~d}, 1 \mathrm{H}, \mathrm{CH}^{\beta}=\mathrm{CH}^{\alpha}\right.$, $J=16.0 \mathrm{~Hz}), 2.39\left(\mathrm{~s}, \mathrm{CH}_{3}, 3 \mathrm{H}\right) ;{ }^{13} \mathrm{C}-\mathrm{NMR}\left(125 \mathrm{MHz}, \mathrm{CDCl}_{3}\right) \delta 200.1,159.0,144.8,130.4,126.4,124.3$, 116.2, 27.2; HR-MS (ES) calcd. for $\mathrm{C}_{10} \mathrm{H}_{10} \mathrm{O}_{2}(\mathrm{M})^{-}:$: 161.0603. Found: 161.0609 .

4-(2', 4'-dihydroxyphenyl)-3(E)-buten-2-one (17). 17 was obtained from 2,4-dihydroxybenzaldehyde (1.44 mmol, $200 \mathrm{mg}$ ) and 1-(triphenylphosphoranylidene)-2-propanone (1.88 mmol, $600 \mathrm{mg}$ ). Reaction was in water $(10.0 \mathrm{~mL})$ at $90{ }^{\circ} \mathrm{C}$ for $2 \mathrm{~h}$. The crude product was purified by column chromatography (EtOAc/hexane, 5:1 $\rightarrow 1: 1)$ to give $78 \%(200 \mathrm{mg}$ ) of $\mathbf{1 7}$ as a bright brown solid; mp. ${ }^{118-120}{ }^{\circ} \mathrm{C}$ decomp., ${ }^{1} \mathrm{H}-\mathrm{NMR}\left(500 \mathrm{MHz}, \mathrm{CD}_{3} \mathrm{OD}\right) \delta 7.91\left(\mathrm{~d}, 1 \mathrm{H}, \mathrm{CH}^{\beta}=\mathrm{CH}^{\alpha}, J=16.0 \mathrm{~Hz}\right)$, $7.39(\mathrm{~d}$, aromatic, $1 \mathrm{H}, J=8 \mathrm{~Hz}), 6.71\left(\mathrm{~d}, 1 \mathrm{H}, \mathrm{CH}^{\beta}=\mathrm{CH}^{\alpha}, J=16.0 \mathrm{~Hz}\right), 6.34-6.32(\mathrm{~m}$, aromatic, $2 \mathrm{H})$, $2.32\left(\mathrm{~s}, \mathrm{CH}_{3}, 3 \mathrm{H}\right) ;{ }^{13} \mathrm{C}-\mathrm{NMR}\left(125 \mathrm{MHz}, \mathrm{CD}_{3} \mathrm{OD}\right) \delta 202.2,162.9,160.4,142.6,131.4,124.1,114.6,109.1$, 103.4, 26.7; HR-MS (ES) calcd. for $\mathrm{C}_{10} \mathrm{H}_{10} \mathrm{O}_{3}(\mathrm{M})^{-}:$: 177.0552. Found: 177.0555.

4-(2', $3^{\prime}, 4^{\prime}$-trihydroxyphenyl)-3(E)-buten-2-one (18). 18 was obtained from 2,3,4-trihydroxybenzaldehyde $(1.29 \mathrm{mmol}, 200 \mathrm{mg}$ ) and 1-(triphenylphosphoranylidene)-2-propanone $(1.94 \mathrm{mmol}, 620 \mathrm{mg})$. Reaction was in water $(10.0 \mathrm{~mL})$ at $90{ }^{\circ} \mathrm{C}$ for $0.5 \mathrm{~h}$. The crude product was purified by column chromatography (EtOAc/hexane, 2:1 $\rightarrow 1: 1)$ to give 70\% (175 mg) of $\mathbf{1 8}$ as a bright brown solid; mp. $98-100{ }^{\circ} \mathrm{C}$ decomp. ${ }^{1} \mathrm{H}-\mathrm{NMR}\left(500 \mathrm{MHz}, \mathrm{CD}_{3} \mathrm{OD}\right) \delta 7.88\left(\mathrm{~d}, 1 \mathrm{H}, \mathrm{CH}^{\beta}=\mathrm{CH}^{\alpha}\right.$, $J=16.1 \mathrm{~Hz}), 6.96(\mathrm{~d}$, aromatic, $1 \mathrm{H}, J=8.7 \mathrm{~Hz}), 6.72\left(\mathrm{~d}, 1 \mathrm{H}, \mathrm{CH}^{\beta}=\mathrm{CH}^{\alpha}, J=16.1 \mathrm{~Hz}\right), 6.37(\mathrm{~d}$, aromatic, $1 \mathrm{H}, J=8.6 \mathrm{~Hz}), 2.33\left(\mathrm{~s}, \mathrm{CH}_{3}, 3 \mathrm{H}\right) ;{ }^{13} \mathrm{C}-\mathrm{NMR}\left(125 \mathrm{MHz}, \mathrm{CD}_{3} \mathrm{OD}\right) \delta 202.1,150.2,148.5,142.9,134.0,124.5$, 121.1, 115.3, 108.7, 26.7; HR-MS (ES) calcd. for $\mathrm{C}_{10} \mathrm{H}_{10} \mathrm{O}_{4}(\mathrm{M})^{-}$: 193.0501. Found: 193.0506. 
4-(3'-methoxy-4'-hydroxyphenyl)-3(E)-buten-2-one (20). $20 \quad$ was obtained from 4-hydroxy-3-methoxybenzaldehyde $(0.65 \mathrm{mmol}, 100 \mathrm{mg})$ and 1-(triphenylphosphoranylidene)-2propanone $(0.98 \mathrm{mmol}, 313 \mathrm{mg})$. Reaction was in water $(4.0 \mathrm{~mL})$ at $90{ }^{\circ} \mathrm{C}$ for $0.5 \mathrm{~h}$. The crude product was purified by column chromatography (EtOAc/hexane, 5:1 $\rightarrow$ 2:1) to give $98 \%(124 \mathrm{mg})$ of 20 as a white solid; mp. 126-128 ${ }^{\circ} \mathrm{C}$ (lit. mp. $126{ }^{\circ} \mathrm{C}$ [39]); ${ }^{1} \mathrm{H}-\mathrm{NMR}\left(500 \mathrm{MHz}, \mathrm{CDCl}_{3}\right) \delta 7.46$ $\left(\mathrm{d}, 1 \mathrm{H}, \mathrm{CH}^{\beta}=\mathrm{CH}^{\alpha}, J=16.0 \mathrm{~Hz}\right), 7.10-7.07(\mathrm{~m}$, aromatic, $1 \mathrm{H}), 7.05(\mathrm{~d}$, aromatic, $1 \mathrm{H}, J=1.5 \mathrm{~Hz})$, $6.92(\mathrm{~d}$, aromatic, $1 \mathrm{H}, J=8 \mathrm{~Hz}), 6.57\left(\mathrm{~d}, 1 \mathrm{H}, \mathrm{CH}^{\beta}=\mathrm{CH}^{\alpha}, J=16.0 \mathrm{~Hz}\right), 6.03(\mathrm{br} \mathrm{s}, \mathrm{OH}, 1 \mathrm{H}), 3.93\left(\mathrm{~s}, \mathrm{CH}_{3}\right.$, 3H), $2.36\left(\mathrm{~s}, \mathrm{CH}_{3}, 3 \mathrm{H}\right) ;{ }^{13} \mathrm{C}-\mathrm{NMR}\left(125 \mathrm{MHz}_{\mathrm{CDCl}}\right) \delta 198.4,148.2,146.9,143.7,126.8,124.9,123.5,114.8$, 109.3, 55.9, 27.2; HR-MS (ES) calcd. for $\mathrm{C}_{11} \mathrm{H}_{12} \mathrm{O}_{3}(\mathrm{M})^{-}:$191.0708. Found: 191.0709.

\subsection{Biological Activities}

\subsubsection{Calculation of $\log P_{\mathrm{o} / \mathrm{w}}$}

The partition coefficient between $n$-octanol and water $\left(\log P_{\mathrm{o} / \mathrm{w}}\right)$ was calculated using the SwissADME web tool [40].

\subsubsection{Determination of the Oxidation Inhibition of the $\mathrm{o} / \mathrm{w}$ Emulsion}

The emulsion containing $15 \mathrm{mg}$ of sorbitan sesquioleate, $50 \mathrm{mg}$ of flaxseed oil, and $2 \mathrm{~mL}$ of $10 \mathrm{mM}$ Tris- $\mathrm{HCl}$ buffer, $\mathrm{pH} 7.4$, was prepared by the method [41,42]. Determination of the oxidation inhibition of the $\mathrm{o} / \mathrm{w}$ emulsion was carried out according to a procedure described previously [43], using $20 \mu \mathrm{L}$ of the 1:1 $(v / v)$ buffer-diluted emulsion, methanolic solution of each compound (final concentration $100 \mu \mathrm{M})$, and ammonium iron(II) sulfate hexahydrate (Mohr's salt) $(5 \mathrm{mM})$. Thiobarbituric acid reacting substances (TBARS) were determined according to [44].

\subsubsection{DPPH Assay}

The 2,2-diphenyl-1-picrylhydrazyl (DPPH) assay was performed using a previously reported method [45] with a modification. A series of samples consisting of $900 \mu \mathrm{L}$ of the $0.04 \mathrm{mg} / \mathrm{mL}$ methanolic solution of DPPH and different concentrations of the compounds (100 $\mu \mathrm{L})$ were prepared. Then, the absorbance at $517 \mathrm{~nm}$ was measured after a $30 \mathrm{~min}$ incubation at room temperature. The half maximal inhibitory concentration $\left(\mathrm{IC}_{50}\right)$ was used as an indicating measure of the scavenging activity.

\subsubsection{Cell Viability Assay in the Normal Human Dermal Fibroblast (NHDF) Cell Line}

The cytotoxicity test was performed as described in [44] using the NHDF cell line treated with the methanolic solution of the tested compounds to obtain the final concentrations of 12.5, 25, 50, 100, 200, or $250 \mu \mathrm{M}$, and incubation for 24 and $48 \mathrm{~h}$. Cell viability was assessed by the MTT method [46].

\section{Conclusions}

To sum up, water is an effective medium for the HWE reaction. A one-step procedure provides $E$-products with a high selectivity and good yields and its application in the synthesis of different phenolic compounds turns out to be easy, fast, and environmentally friendly. The synthetic method for the preparation of phenolic caffeic acid derivatives in water is advantageous because of the mild reaction conditions and the ease of manipulation. The most important advantage of this procedure, in comparison to the methods with the protected phenolic groups, is the elimination of environmentally unsafe reagents (e.g., $\mathrm{BBr}_{3}$ ) and harsh reaction conditions. Moreover, the development of an efficient HWE process broadens the spectrum of the synthetic polyphenols available for potential use in the pharmaceutical or cosmetic industries. The obtained analogues of caffeic acid were tested for their antioxidant activity, and three of them $(\mathbf{1 2}, \mathbf{1 3}$, and 18) displayed a higher antioxidant activity than caffeic acid. Exhaustive biological studies of the anti-inflammatory, antibacterial, and antiviral activity are to be the subject of further investigation. 
Supplementary Materials: The supplementary materials are available online.

Author Contributions: K.S., concept, design, performing the synthetic research, analyzing the data, and writing the manuscript. A.J. and N.F., biological investigation, analyzing the data, writing the manuscript. P.C., performing the spectroscopy experiments. M.C., contribution to the discussion of the synthetic results and writing of the manuscript.

Funding: This research received no external funding.

Acknowledgments: This work was supported under the framework of a statutory project funded by the Polish Ministry of Science and Higher Education (Pharmaceutical Research Institute) and by a statutory activity of subsidy from the Polish Ministry of Science and Higher Education for the Faculty of Biotechnology of University of Wroclaw. Publication costs were supported by Wroclaw Center of Biotechnology program "The Leading National Research Center (KNOW) for years 2014-2018".

Conflicts of Interest: The authors have declared that no competing interests exist.

\section{Abbreviations}

$\begin{array}{ll}\text { CA } & \text { caffeic acid } \\ \text { HWE } & \text { Horner-Wadsworth-Emmons } \\ \text { DCM } & \text { dichloromethane } \\ \text { DPPH } & \text { 2,2-diphenyl-1-picrylhydrazyl } \\ \text { EtOAc } & \text { ethyl acetate } \\ \text { DMSO } & \text { dimethyl sulfoxide } \\ \log P_{\text {o/w }} & \text { partition coefficient between } n \text {-octanol and water } \\ \text { MTT } & \text { 3-(4,5-dimethyl-2-thiazolyl)-2,5-diphenyl-2H-tetrazolium bromide } \\ \text { NHDF } & \text { normal human dermal fibroblasts } \\ \text { TBARS } & \text { thiobarbituric acid reactive substances }\end{array}$

\section{References}

1. Shahidi, F.; Yeo, J. Bioactivities of phenolics by focusing on suppression of chronic diseases: A review. Int. J. Mol. Sci. 2018, 19, 1573. [CrossRef] [PubMed]

2. Bernini, R.; Gilardini Montani, M.S.; Merendino, N.; Romani, A.; Velotti, F. Hydroxytyrosol-derived compounds: A basis for the creation of new pharmacological agents for cancer prevention and therapy. J. Med. Chem. 2015, 58, 9089-9107. [CrossRef] [PubMed]

3. Zhang, P.; Tang, Y.; Li, N.G.; Zhu, Y.; Duan, J.A. Bioactivity and chemical synthesis of caffeic acid phenethyl ester and its derivatives. Molecules 2014, 19, 16458-16476. [CrossRef] [PubMed]

4. Quideau, S.; Deffieux, D.; Douat-Casassus, C.; Pouysegu, L. Plant polyphenols: Chemical properties, biological activities, and synthesis. Angew. Chem. Int. Ed. 2011, 50, 586-621. [CrossRef] [PubMed]

5. Gomes, C.A.; Girao da Cruz, T.; Andrade, J.L.; Milhazes, N.; Borges, F.; Marques, M.P.M. Anticancer activity of phenolic acids of natural or synthetic origin: A structure-activity study. J. Med. Chem. 2003, 46, 5395-5401. [CrossRef] [PubMed]

6. Eid, H.M.; Vallerand, D.; Muhammad, A.; Durst, T.; Haddad, P.S.; Martineau, L.C. Structural constraints and the importance of lipophilicity for the mitochondrial uncoupling activity of naturally occurring caffeic acid esters with potential for the treatment of insulin resistance. Biochem. Pharmacol. 2010, 79, 444-454. [CrossRef] [PubMed]

7. Takahashi, K.; Yoshioka, Y.; Kato, E.; Katsuki, S.; Iida, O.; Hosokawa, K.; Kawabata, J. Methyl caffeate as an $\alpha$-glucosidase inhibitor from Solanum torvum fruits and the activity of related compounds. Biosci. Biotechnol. Biochem. 2010, 74, 741-745. [CrossRef] [PubMed]

8. Ganugapati, J.; Swarna, S. Molecular docking studies of antidiabetic activity of cinnamon compounds. Asian J. Pharm. Clin. Res. 2014, 7, 31-34.

9. Fiuza, S.M.; Gomes, C.; Teixeira, L.J.; Girao da Cruz, M.T.; Cordeiro, M.N.D.S.; Milhazes, N.; Borgesa, F.; Marques, M.P.M. Phenolic acid derivatives with potential anticancer properties-A structure-activity relationship study. Part 1: Methyl, propyl and octyl esters of caffeic and gallic acids. Bioorg. Med. Chem. 2004, 12, 3581-3589. [CrossRef] [PubMed] 
10. Monteiro, L.S.; Oliveira, S.; Paiva-Martins, F.; Ferreira, P.M.T.; Pereira, D.M.; Andrade, P.B.; Valentao, P. Synthesis and preliminary biological evaluation of new phenolic and catecholic dehydroamino acid derivatives. Tetrahedron 2017, 73, 6199-6209. [CrossRef]

11. Chen, J.H.; Chi-Tang, H. Antioxidant activities of caffeic acid and its related hydroxycinnamic acid compounds. J. Agric. Food Chem. 1997, 7, 2374-2378. [CrossRef]

12. Sato, Y.; Itagaki, S.; Kurokawa, T.; Ogura, J.; Kobayashi, M.; Hirano, T.; Sugawara, M.; Iseki, K. In vitro and in vivo antioxidant properties of chlorogenic acid and caffeic acid. Int. J. Pharm. 2010, 403, 136-138. [CrossRef] [PubMed]

13. Losada-Barreiro, S.; Bravo-Díaz, C. Free radicals and polyphenols: The redox chemistry of neurodegenerative diseases. Eur. J. Med. Chem. 2017, 133, 379-402. [CrossRef] [PubMed]

14. Srivastava, V.; Darokar, M.P.; Fatima, A.; Kumar, J.K.; Chowdhury, C.; Saxena, H.O.; Dwivedi, G.R.; Shrivastava, K.; Gupta, V.; Chattopadhyay, S.K.; et al. Synthesis of diverse analogues of Oenostacin and their antibacterial activities. Bioorg. Med. Chem. 2007, 15, 518-525. [CrossRef] [PubMed]

15. Siquet, C.; Paiva-Martins, F.; Lima, J.L.F.C.; Reis, S.; Borges, F. Antioxidant profile of dihydroxy- and trihydroxyphenolic acids- A structure-activity relationship study. Free Radic. Res. 2006, 40, 433-442. [CrossRef] [PubMed]

16. Kuo, P.C.; Damu, A.G.; Cherng, C.Y.; Jeng, J.F.; Teng, C.M.; Lee, E.J.; Wu, T.S. Isolation of a natural antioxidant, dehydrozingerone from Zingiber officinale and synthesis of its analogues for recognition of effective antioxidant and antityrosinase agents. Arch. Pharm. Res. 2005, 28, 518-528. [CrossRef] [PubMed]

17. Alvarado, I.E.; Navarro, D.; Rocord, E.; Asther, M. Fungal biotransformation of p-coumaric acid into caffeic acid by Pycnoporus cinnabarinus: An alternative for producing a strong natural oxidant. World J. Microbiol. Biotechnol. 2003, 19, 157-160. [CrossRef]

18. Kroon, P.A.; Williamson, G. Hydroxycinnamates in plants and food: Current and future perspectives. J. Sci. Food Agric. 1999, 79, 355-361. [CrossRef]

19. Touaibia, M.; Jean-François, J.; Doiron, J. Caffeic acid, a versatile pharmacophore: An overview. Mini Rev. Med. Chem. 2011, 11, 695-713. [CrossRef] [PubMed]

20. Furuya, T.; Arai, Y.; Kino, K. Biotechnological production of caffeic acid by bacterial cytochrome P450 CYP199A2. Appl. Environ. Microbiol. 2012, 78, 6087-6094. [CrossRef] [PubMed]

21. Jafari, A.A.; Ghadami, M. Efficient synthesis of $\alpha, \beta$-unsaturated ketones with trans-selective Horner-Wadsworth-Emmons reaction in water. Environ. Chem. Lett. 2016, 14, 223-228. [CrossRef]

22. Wadsworth, W.S. Synthetic applications of phosphoryl-stabilized anions. Org. React. 2005, 25, 73-253.

23. Wadsworth, W.S.; Emmons, W.D. The utility of phosphonate carbanions in olefin synthesis. J. Am. Chem. Soc. 1961, 83, 1733-1738. [CrossRef]

24. Ando, K. Z-Selective Horner-Wadsworth-Emmons reaction of $\alpha$-substituted ethyl (diarylphosphono)acetates with aldehydes. J. Org. Chem. 1998, 63, 8411-8416. [CrossRef]

25. Saito, N.; Ryoda, A.; Nakanishi, W.; Kumamoto, T.; Ishikawa, T. Guanidine-catalyzed asymmetric synthesis of 2,2-disubstituted chromane skeletons by intramolecular oxa-Michael addition. Eur. J. Org. Chem. 2008, 2759-2766. [CrossRef]

26. Patil, V.J.; Mavers, U. Wittig reactions in the presence of Silica gel. Tetrahedron Lett. 1996, 37, $1281-1284$. [CrossRef]

27. Isaacs, N.S.; El-Din, G.N. The application of high pressure to some difficult Wittig reactions. Tetrahedron Lett. 1987, 28, 2191-2192. [CrossRef]

28. Hooper, D.L.; Garagan, S.; Kayser, M.M. Lithium cation-catalyzed Wittig reactions. J. Org. Chem. 1994, 59, 1126-1128. [CrossRef]

29. Boulaire, V.L.; Gree, R. Wittig reactions in the ionic solvent [bmim][BF 4 . Chem. Commun. 2000, 22, 2195-2196. [CrossRef]

30. Spinella, A.; Fortunati, T.; Soriente, A. Microwave accelerated Wittig reactions of stabilized phosphorus ylides with ketones under solvent-free conditions. Synlett 1997, 1, 93-94. [CrossRef]

31. Dambacher, J.; Zhao, W.; El-Batta, A.; Anness, R.; Jiang, C.; Bergdahl, M. Water is an efficient medium for Wittig reactions employing stabilized ylides and aldehydes. Tetrahedron Lett. 2005, 46, 4473-4477. [CrossRef]

32. El-Batta, A.; Jiang, C.; Zhao, W.; Anness, R.; Cooksy, A.L.; Bergdahl, M. Wittig reactions in water media employing stabilized ylides with aldehydes. Synthesis of $\alpha, \beta$-unsaturated esters from mixing aldehydes, $\alpha$-bromoesters, and $\mathrm{Ph}_{3} \mathrm{P}$ in aqueous $\mathrm{NaHCO}_{3}$. J. Org. Chem. 2007, 72, 5244-5259. [CrossRef] [PubMed] 
33. Nonnenmacher, A.; Mayer, R.; Plieninger, H. Application of high-pressure on Wittig reactions with resonance stabilized ylides. Liebigs Ann. Chem. 1983, 12, 2135-2140. [CrossRef]

34. Saija, A.; Tomaino, A.; Lo Cascio, R.; Trombetta, D.; Proteggente, A.; De Pasquale, A.; Uccela, N.; Bonina, F. Ferulic and caffeic acids as potential protective agents against photooxidative skin damage. J. Sci. Food Agric. 1999, 79, 476-480. [CrossRef]

35. Saija, A.; Tomaino, A.; Trombetta, D.; de Pasquale, A.; Uccella, N.; Barbuzzi, T.; Paolino, D.; Bonina, F. In vitro and in vivo evaluation of caffeic and ferulic acids as topical photoprotective agents. Int. J. Pharm. 2000, 199, $39-47$. [CrossRef]

36. Pluemsamran, T.; Onkoksoong, T.; Panich, U. Caffeic acid and ferulic acid inhibit UVA-induced matrix metalloproteinase-1 through regulation of antioxidant defense system in keratinocyte HaCaT cells. Photochem. Photobiol. 2012, 88, 961-968. [CrossRef] [PubMed]

37. Huang, W.J.; Chen, C.C.; Chao, S.W.; Lee, S.S.; Hsu, F.L.; Lu, Y.L.; Hung, M.F.; Chang, C.I. Synthesis of N-hydroxycinnamides capped with a naturally occurring moiety as inhibitors of histone deacetylase. Chem. Med. Chem. 2010, 5, 598-607. [CrossRef] [PubMed]

38. Viviano, M.; Glasnov, T.N.; Reichart, B.; Tekautz, G.; Kappe, C.O. A scalable two-step continuous flow synthesis of nabumetone and related 4-Aryl-2-butanones. Org. Process Res. Dev. 2011, 15, 858-870. [CrossRef]

39. Oh, S.; Jang, S.; Kim, D.; Han, I.O.; Jung, J.Ch. Synthesis and evaluation of biological properties of benzylideneacetophenone derivatives. Arch. Pharm. Res. 2006, 29, 469-475. [CrossRef] [PubMed]

40. Daina, A.; Michielin, O.; Zoete, V. SwissADME: A free web tool to evaluate pharmacokinetics, drug-likeness and medicinal chemistry friendliness of small molecules. Sci. Rep. 2017, 7, 42717. [CrossRef] [PubMed]

41. Zarnowski, R.; Jaromin, A.; Certik, M.; Czabany, T.; Fontaine, J.; Jakubik, T.; Iqbal, M.C.M.; Grandmougin-Ferjani, A.; Kozubek, A.; Pietr, S.J. The oil of Adenanthera pavonina L. seeds and its emulsions. Z. Naturforsch. C. 2004, 59, 321-326. [CrossRef] [PubMed]

42. Jaromin, A.; Zarnowski, R.; Kozubek, A. Emulsions of oil from Adenanthera pavonina L. seeds and their protective effect. Cell Mol. Biol. Lett. 2006, 11, 438-448. [CrossRef] [PubMed]

43. Jaromin, A.; Zarnowski, R.; Piętka-Ottlik, M.; Andes, D.R.; Gubernator, J. Topical delivery of ebselen encapsulated in biopolymeric nanocapsules: Drug repurposing enhanced antifungal activity. Nanomedicine 2018, 13, 1139-1155. [CrossRef] [PubMed]

44. Buege, J.A.; Aust, S.D. Microsomal lipid peroxidation. Methods Enzymol. 1978, 52, 302-310. [PubMed]

45. Zhang, Z.; Xiao, B.; Chen, Q.; Lian, X.Y. Synthesis and biological evaluation of caffeic acid 3,4-dihydroxyphenethyl ester. J. Nat. Prod. 2010, 73, 252-254. [CrossRef] [PubMed]

46. Mosmann, T. Rapid colorimetric assay for cellular growth and survival: Application to proliferation and cytotoxicity assays. J. Immunol. Methods 1983, 65, 55-63. [CrossRef]

Sample Availability: Samples of the compounds are not available from the authors.

(c) 2018 by the authors. Licensee MDPI, Basel, Switzerland. This article is an open access article distributed under the terms and conditions of the Creative Commons Attribution (CC BY) license (http:/ / creativecommons.org/licenses/by/4.0/). 